\title{
Variable moduli of soil strain
}

\author{
Karim Sultanov ${ }^{1, *}$, Pavel Loginov ${ }^{2}$, Sabida Ismoilova $^{2}$ and Zulfiya Salikhova ${ }^{2}$ \\ ${ }^{1}$ Tashkent Institute Irrigation and Agricultural Mechanization Engineers \\ ${ }^{2}$ Institute of Mechanics and Seismic Stability of Structures named after M.T. Urazbayev of the \\ Academy of Sciences of the Republic of Uzbekistan
}

\begin{abstract}
The experimental diagrams between stress and strain components for soft soils are non-linear. Nonlinear diagrams qualitatively differ for soils of undisturbed and disturbed structures. It is believed that the manifestations of nonlinear properties of soil are associated with microdestruction of soil structure under compression and, therefore, with changes in its mechanical characteristics under strain. It follows that the modulus of elasticity, Poisson's ratio, viscosity and other mechanical parameters are the variables in the process of soil strain. Based on this, from the experimental results given in scientific literature, the changes in the modulus of elasticity and plasticity of soil are determined depending on the values of compression strain. In the process of static and dynamic compression of soil it is almost impossible to determine the boundaries of elastic and plastic strains in soft soil. So, the modulus under soil compression is called the strain modulus. From published results of experiments on dynamic and static compression of soil the most informative ones have been selected. Processing the selected compression diagrams of soft soil, the secant moduli of strain for loess soil and clay have been determined. It is established that the moduli of strain of clay and loess soil under static and dynamic strain vary depending on the rate of strain, the state of the structure and the level of compressive load.
\end{abstract}

\section{Introduction}

Determination and assessment of reliability (stability, seismic resistance) in computation of aboveground and underground structures require the knowledge of strength and mechanical characteristics of soil. Strength characteristics of soil, as is well known, mainly include the coefficient of adhesion and the angle of internal friction. Mechanical characteristics of soil are: the modulus of elasticity, Poisson's ratio, modulus of unloading, coefficient of soil viscosity, etc. Strength and mechanical characteristics of soils are determined experimentally.

Strength characteristics of soil and rocks under static loads are experimentally defined in [1-3]. In [1], strength characteristics of loess soil stabilized by silica nanoparticles are determined. It is shown that in this case strength characteristics of soil increase. In [2], strength characteristics of soil are determined depending on water saturation using a flat cut method and a triaxial compression method. In [3], strength characteristics of soil are defined in relation to the stability of slopes.

\footnotetext{
* Corresponding author: sultanov.karim@mail.ru
} 
In $[4,3]$, strength characteristics of soil under dynamic loads are experimentally determined in field conditions. In [4], the resistance of soft clay to shear is determined by the free fall of a steel sphere with a diameter of $0.25 \mathrm{~m}$ into water. The measuring unit located inside the sphere, measures the penetration into soil. The obtained acceleration measurement data allows determining strength characteristics of soil. In [5], by a similar method, the strength characteristics of the seabed are determined at different rates of a steel ball impact.

Experimental determination of mechanical characteristics of soil under static loads is the subject of study in [6-9]. In [6], elastic modulus of granular material is determined at low levels of strain, depending on the diameter of the grain particles. It has been established that the Young's modulus is practically independent on the diameter of particles of granular material. In [7], the strain moduli are determined for compression and die testing of soil. Strain characteristics of soil under triaxial cyclic loading are defined in [8]. Mechanical viscoelastic characteristics of geosynthetic material under static stresses are defined in [9].

Mechanical characteristics of soil under dynamic loads are also defined in [10-16]. In [10], mechanical characteristics of brittle rocks are determined at load rates of axial stress from 0.05 to $10 \mathrm{kN} / \mathrm{s}$ in laboratory conditions. It is established that the modulus of elasticity of the rock mass increases with increasing of load rate. In [11], elastic properties of clay soil are determined by measuring the velocities of ultrasonic waves of various frequencies in soil. Here, the experimentally obtained dynamic modulus is compared with the static modulus. In [12], mechanical characteristics of soft clay are determined under dynamic loads by the centrifuge simulation method.

In the studies considered above, mechanical characteristics of soil are determined by indirect methods, without constructing soil compression diagrams $\sigma_{i j}\left(\varepsilon_{i j}\right)$, where $\sigma_{i j}$-are the components of the stress tensor, and $\varepsilon_{i j}$ - the corresponding components of the strain tensor. In [13-16] the results of extensive laboratory and field experimental studies of the compressibility of various soils under static and dynamic loads are presented. In [13-16], static experiments have been carried out in the laboratory, and dynamic experiments in laboratory and field conditions. Static loads have been created using ordinary presses on special installations. Dynamic loads have been initiated by the explosion of TNT charges in field conditions or by dropping a load of a certain weight into a special installation, with the soil sample placed inside. A description of the special facilities created for the experiments and the method of soil samples testing are described in detail in [13-16]. According to the results of experiments, a number of diagrams of volume compression of soil $\sigma_{1}(\varepsilon)$ are constructed, where, $\sigma_{1}=-p$ is the volume stress, $p-$ is the pressure, $\varepsilon-$ is the volume strain.

As shown by the results of experiments given in [13-16], the diagrams $\sigma_{1}(\varepsilon)$ are nonlinear.

In [16-19], the manifestation of nonlinear properties of soil strain is explained by the destruction of soil structure under compression. Consequently, physico-mechanical and mechanical characteristics of soil change as well. Based on this hypothesis, nonlinear laws of strain of soft soil are constructed in [16-19]. Using nonlinear models of soil strain given in [20-23], some problems of seismic stability of underground and aboveground structures are solved.

The nonlinear laws proposed in [16-19] include nonlinear dependences $E(\varepsilon)$, where $E-$ is the modulus of volume strain of soil, which varies under soil compression, depending on the values of strain. 
Nonlinear functions $E(\varepsilon)$ can only be determined from experimental diagrams $\sigma_{1}(\varepsilon)$. Note that in the diagrams $\sigma_{1}(\varepsilon)$ given in [13-16], it is impossible to explicitly determine the boundaries of elastic and plastic strain of soil. Therefore, the modulus of elasticity and the modulus of plasticity are called by one term - the modulus of strain.

The aim of this work is to determine non-linear functions $E(\varepsilon)$ for different types of soil under static and dynamic loads from experimental diagrams $\sigma_{1}(\varepsilon)$ given in [13-16].

\section{Methods}

According to $[16,17]$, the nonlinearity of dependences $\sigma_{1}(\varepsilon)$ is associated with a change in the soil structure under compression. When soil is compressed, a complex process of its structure change occurs, associated with mutual relative displacements of solid particles of soil; this process primarily determines the nature of the change in dependence $\sigma_{1}(\varepsilon)$, that is, the patterns of soil compression. A change in soil structure (destruction, restructuring, repacking) may lead to a change in physical and mechanical properties of soil. This means that under compression, the soil structure changes in the process of strain and, as a result, its initial mechanical characteristics also change: the density, elastic modulus, Poisson's ratio, viscosity, etc.

Typical curves of compressive force dependence on relative strain are given in [17]. According to [17], there are three types of the curves, observed in experiments [13-16]. Experimental diagrams [13-16] make it possible to determine the change in the current modulus of soil strain under compression. In the process of compression, soil undergoes simultaneously elastic and plastic strain stages [17], therefore, elastic modulus hereinafter is called the strain modulus.

From the diagram $\sigma_{1}(\varepsilon)$ one can determine the actual strain modulus $E_{f}$ or the secant strain modulus $E$.

To determine $E$ the curve $\sigma_{1}(\varepsilon)$ is divided into 50 or more intervals with approximately the same step $\Delta \varepsilon$. Further, the values $\Delta \sigma$ corresponding to $\Delta \varepsilon$ and $E_{f}=\Delta \sigma / \Delta \varepsilon$ are determined. The value of the secant strain modulus $E$ for the same interval points is defined as $E=\sigma_{1} / \varepsilon$, where $\sigma_{1}$ and $\varepsilon-$ are the values of longitudinal compressive (volume) force and the strain at the boundaries of the intervals, respectively.

As shown by the results of processing experimental diagrams $\sigma_{1}(\varepsilon)$, changes in the secant modulus of strain $E$ with an increase in compressive strain $\varepsilon$, occur smoothly and steadily. Changes in the actual modulus of strain $E_{f}$ occur irregularly and with jumps. Therefore, the secant strain modulus $E$ (hereinafter the strain modulus) is determined from the experimental compression diagrams $\sigma_{1}(\varepsilon)$.

\section{Results}

Experimental diagrams are taken from $[13,15,16]$. The choice of experimental dependences from the numerous results of the experiments given in $[13,15,16]$ is random. Figure 1 shows the diagrams for a clay sample of undisturbed structure under dynamic (curves 1 and 2) and static (curve 3) loads [15]. The experiments have been conducted on a special device 
in the laboratory. The method of conducting experiments and extracting a sample of clay of undisturbed structure and its preparation for the experiment are described in detail in [15].

Samples of clay had the following physico-mechanical characteristics: $\rho_{0}=1700-1750 \mathrm{~kg} / \mathrm{m}^{3}$ and $W=20-22 \%$ where $\rho_{0}$ - is the initial density of clay sample, $W-$ is the gravimetric moisture.

In Figure 1, curve 0 is theoretically obtained, i.e. this is a compression diagram of clay obtained by calculation in [15] at a strain rate $\mathrm{d} \varepsilon / \mathrm{d} t \rightarrow \infty$. Curve 1 is experimentally obtained at the first compression of soil under the impact of a load of $1 \mathrm{kN}$ in weight. The height of the load fall is $0.25 \mathrm{~m}$. The impact was made through the cushion, which softens the dynamic load. Strain rate at loading for curve 1 is $\mathrm{d} \varepsilon / \mathrm{d} t=20 \mathrm{~s}^{-1}$. Curve 2 refers to the repeated loading of the same clay sample, under repeated impact of the same parameters as the first one. In this case we get $\mathrm{d} \varepsilon / \mathrm{d} t=35 \mathrm{~s}^{-1}$. Curve 3 is obtained by static loading of another clay sample of undisturbed structure. For curve 3 we get $\mathrm{d} \varepsilon / \mathrm{d} t \rightarrow 0$.

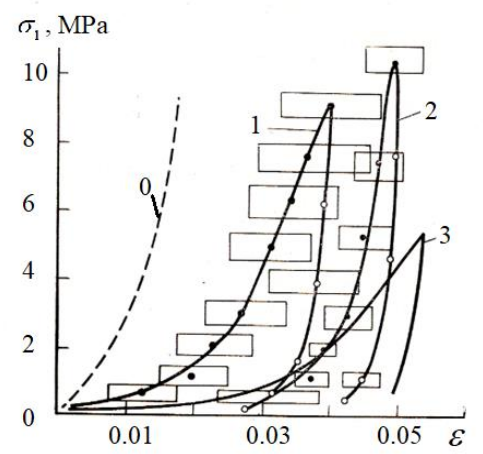

Fig. 1. Experimental diagrams of clay compression [15] under dynamic (curves $1,2)$ and static (curve 3 ) loads

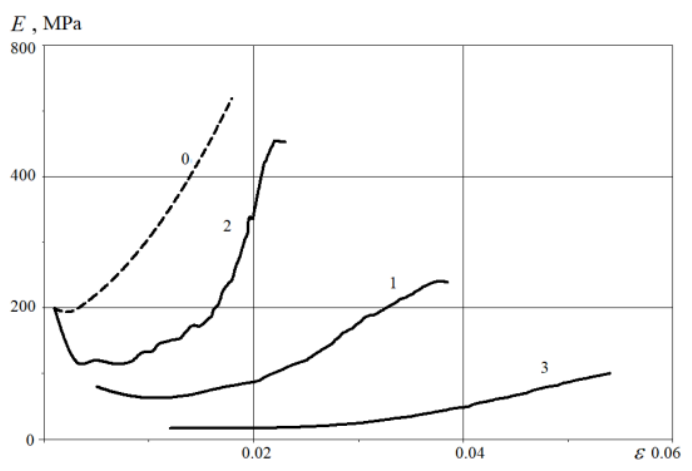

Fig. 2. Change in secant modulus of strain of clay under compression

Changes in the strain moduli determined using curves 0-3 (Figure 1) are shown in Figure 2 (curves 0-3). As seen from Figure 2, changes in the modulus of strain of clay sample significantly depend on the rate of strain $\mathrm{d} \varepsilon / \mathrm{d} t$ or the rate of dynamic loading. With an increase in strain at strain rates not equal to zero, the values of the strain modulus at the initial process of compression decrease. This is due to the destruction of soil structure at the initial stage of dynamic compression. Then, the value of $E$ increases as the soil compresses. Under static compression of soil, at $\mathrm{d} \varepsilon / \mathrm{d} t \approx 0$, the modulus of strain monotonously increases. Apparently, in this case, structural damage (micro-cracks) under static compression has time to "heal" and the strain modulus grows as the density of soil increases.

The maximum load under static and dynamic compression of clay sample varies from 6 to $10 \mathrm{MPa}$. The clay sample takes no more than $5 \%$ of the volume strain $\left(\varepsilon_{\max }=0.05\right)$. The moduli of strain in this case vary from 10 to $100 \mathrm{MPa}$ under static loading. Under dynamic loading they vary from $100 \mathrm{MPa}$ to $600 \mathrm{MPa}$, depending on the strain rate.

As the results, Figure 2, show, the modulus of clay strain under compression changes significantly.

Figure 3 shows the dynamic compression diagrams of loess soil of the disturbed structure at the initial density of $\rho_{0}=1370-1450 \mathrm{~kg} / \mathrm{m}^{3}$ and humidity $W=14 \%$, taken from [14]. Dynamic load has been created by an explosion in the field site. The technique 
of the experiments is described in [16]. In Figure 3, curves 1-5 refer to the distances from the initial section equal to $0.2 ; 0.3 ; 0.4 ; 0.5$ and $0.8 \mathrm{~m}$, respectively. The values of the strain rate $\mathrm{d} \varepsilon / \mathrm{d} t=100,40,15,10,2 \mathrm{~s}^{-1}$ correspond to curves $1-5$. Changes in the strain moduli with an increase in the volume strain under loading, i.e. dependencies $E(\varepsilon)$ for curves 1-5 (Figure 3) are shown in Figure 4.

As seen from Figure 4, for loess soil of disturbed structure, the values of the strain modulus with increasing strain for curves 1 and 2 continuously increase. Due to the disturbance of the structure under compression, the soil immediately begins to compact and the value of $E$ increases. The values of $E$ in this case also significantly depend on the strain rate under loading. For large values of the strain rate, when $\mathrm{d} \varepsilon / \mathrm{d} t=100$ and 40 (curves 1 and 2), this is significantly noticeable. At low strain rates, changes in $E$ at increasing strain are less noticeable.

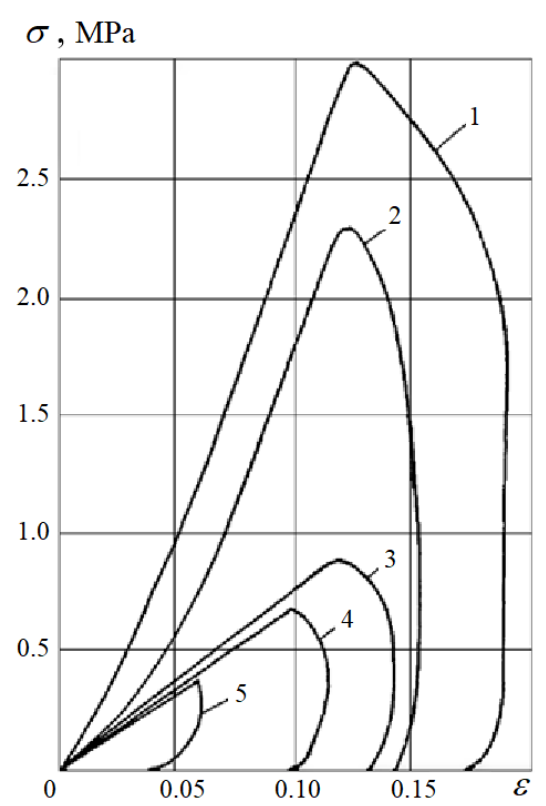

Fig. 3. Experimental dynamic compression diagrams of loess soil [16]

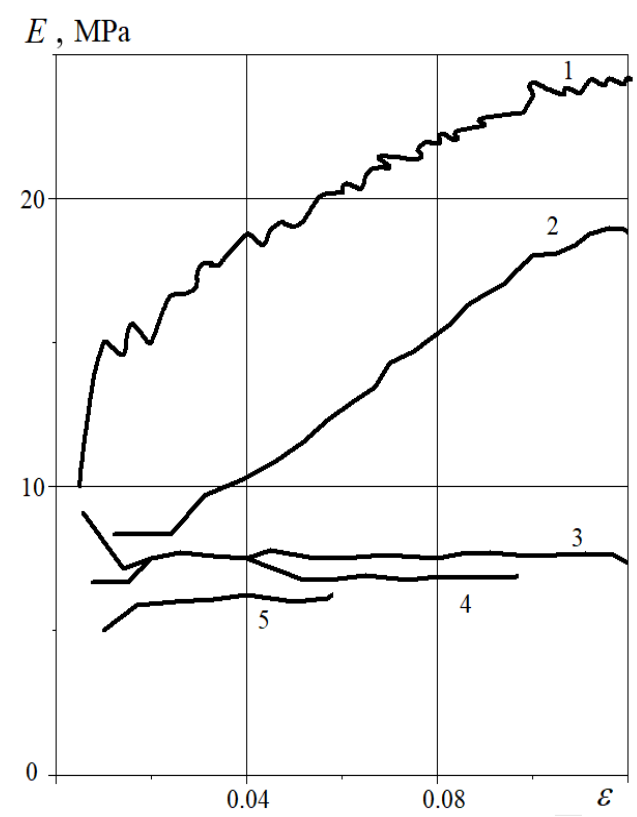

Fig. 4. Changes in secant modulus of strain of loess soil under loading

The static compression diagrams [13] of loess soil with an initial density of $\rho_{0}=1500$ $\mathrm{kg} / \mathrm{m}^{3}$ and humidity $W=10.8 \%$ obtained in laboratory conditions are shown in Figure 5 . The experiments in this case have been carried out using a different technique, described in detail in [13].

Curves 1 and 2 in Figure 5 relate to different experiments with different samples of loess soil of undisturbed structure. In experiments, the static load reached 0.12-0.17 $\mathrm{MPa}$, i.e. was not high. Under these loads, the volume strain changed by $3 \%$.

Changes in the strain modulus in this case are shown in Figure 6. As seen from Figure 6 , under static compression of soil, at $\mathrm{d} \varepsilon / \mathrm{d} t \rightarrow 0$ the strain modulus monotonically increases with strain. 


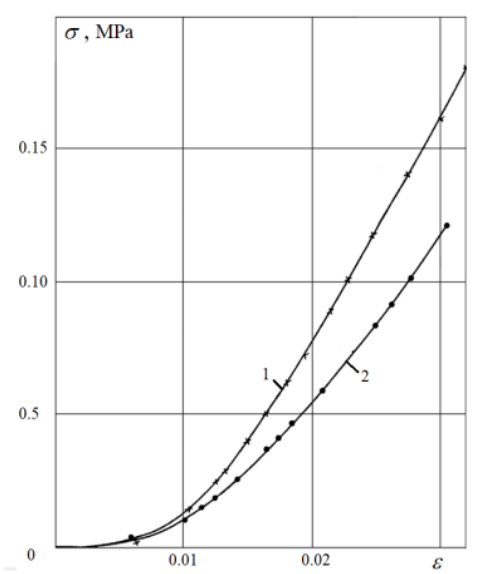

Fig. 5. Compression diagrams of loess soil under dynamic loads [13]

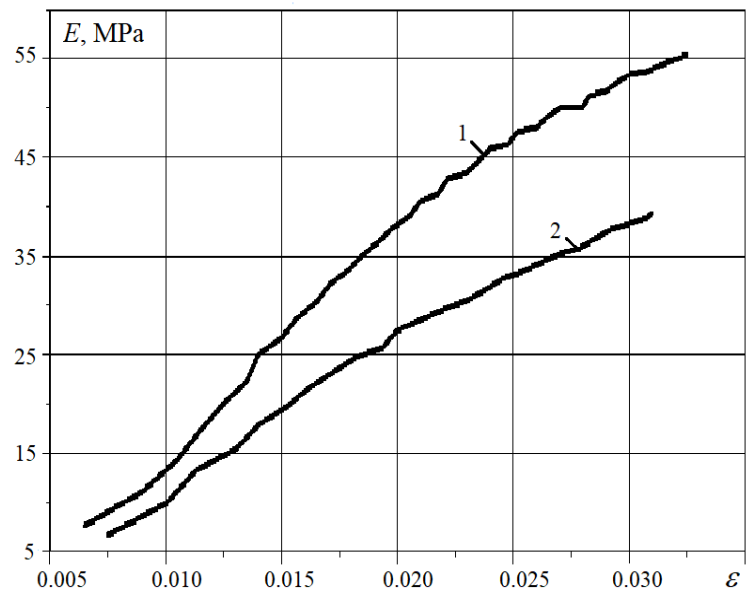

Fig. 6. Change in strain modulus of loess soil under loading

A sample of loess soil with an initial density of $\rho_{0}=1400 \mathrm{~kg} / \mathrm{m}^{3}$ and humidity $W=5.6 \%$, [13], is subjected to cyclic static loading in laboratory conditions (Figure 7). The first half-cycle of loading is carried out up to $0.5 \mathrm{MPa}$, the second - up to $1.0 \mathrm{MPa}$, the third - up to 2.0 MPa and the fourth - up to 3.0 MPa. In all half-cycles, the value of $E$ determined from the diagrams in Figure 7 increases with increasing strain (Figure 8). With an increase in the density of soil, at significant strains equal to 6 and $10 \%$, the values of $E$ remain approximately constant (Figure 8). The range of variation of the volume compression modulus of loess soil under static compression, according to the results in Figure 8, is approximately from 5 to $25 \mathrm{MPa}$.

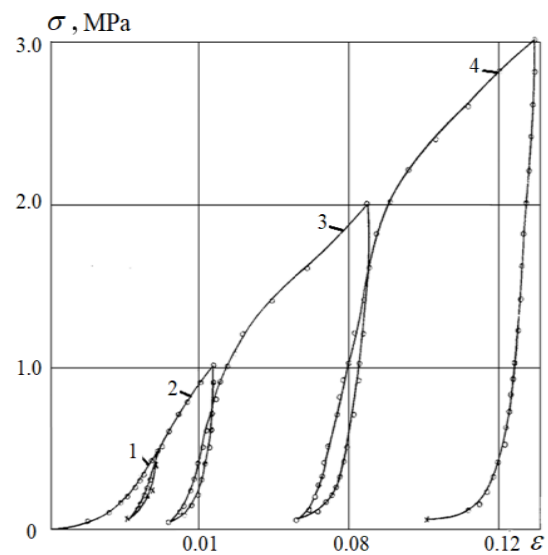

Fig. 7. Compression diagrams of loess soil under half-cyclic static loads [13]

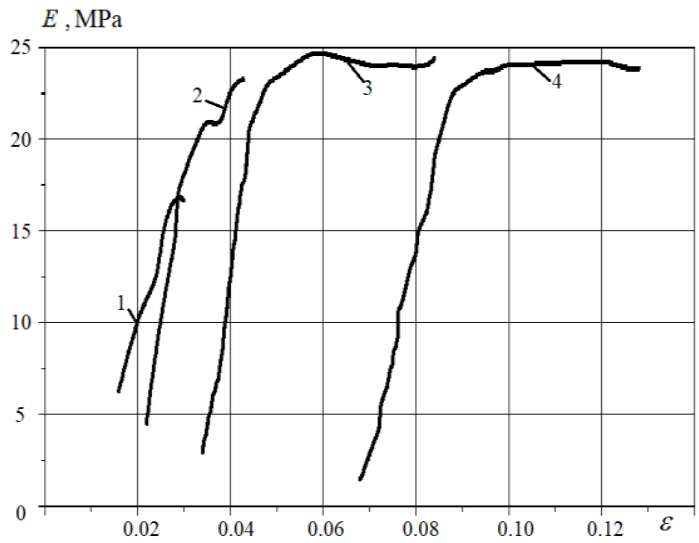

Fig. 8. Change in strain modulus of loess soil under half-cyclic static loads

\section{Discussion}

Processing the results of static and dynamic experiments $[13,15,16]$ on soil compression, Figures 2, 4, 6 and 8, show that the modulus of volume strain of loess soil and clay under 
compression vary significantly. The change in the strain modulus in these cases is up to 5 times or more. Besides, this change in strain modulus in some cases, for example, for clay (Figure 2) is rather curvilinear. The change in strain modulus also significantly depends on the strain rate. As the strain rate increases at the same strain value, the strain modulus values are always greater.

The results of processing a set of diagrams given in $[13,15,16]$ have shown that the change in function $E(\varepsilon)$ is quite complex and it depends on many factors, such as:

strain rate under loading;

soil moisture content;

the state of soil structure (disturbed or undisturbed structure);

maximum load value;

soil types.

The problem ahead is to establish the single-value relationships for changes in the modulus of volume strain and other mechanical characteristics of soils (shear modulus, viscosity coefficient, etc.) based on the above factors.

Next, it is necessary to analytically describe these changes using the simplest functions that could be used in the equations of state of soil. Analytical description of function $E(\varepsilon)$ shown in Figures 2, 4, 6 and 8 may lead to the creation of new physically nonlinear laws of strain of various types of soil.

Note that the change in the strain modulus is determined only at the stage of soil loading under static and dynamic loads. It is also necessary to determine the changes in the strain modulus at the stage of unloading, which is no less interesting.

Thus, the results of processing known experimental data show that under soil strain, its modulus of strain significantly changes. An account of this factor leads to a more fundamental understanding of the processes of strain and unloading in soil. This circumstance is essential in design of buildings and structures for stability, reliability and seismic resistance.

\section{Conclusions}

1. Based on known compression diagrams, the changes in the modulus of volume strain of loess soil and clay are determined in the process of their dynamic and static compression.

2. Graphic dependence of the changes in strain modulus on the strain rate, structural state (disturbed or undisturbed structure), degree of moisture content and the type of soil is established.

3. The variability of the strain modulus in the process of soil strain and their analytical description may lead to the creation of new physically nonlinear laws of strain of soft soil, being the basis for developing new, more advanced methods for calculating buildings and structures for reliability, stability and seismic resistance.

\section{References}

1. L.V. Qingfend, Chengrui Chang, Benhai Zhao, Bo Ma Loess, Soil Mechanics and Foundation Engineering, 54, 6 (2017)

2. N.P. Zung, Magazine of Civil Engineering 9, 35 (2012)

3. A.L. Goldin, Nguyen Phuong Zung, Magazine of Civil Engineering, 9, 35 (2012)

4. J.P. Morton, C.D. O'Loughlin, D.J. White, Géotechnique, 66, 12 (2016)

5. S.H. Chow, D.W. Airey, Géotechnique, 63, 13 (2013)

6. T. Enomoto, Soils and Foundations, 56, 4 (2016) 
7. O.I. Ignatova, Soil Mechanics and Foundation Engineering, 5 (2009)

8. A.S. Alexandrov, Magazine of Civil Engineering, 5 (2013)

9. I.I. Loginova, D.A. Artamonova, O.N. Stolyarov, B.E. Melnikov, Magazine of Civil Engineering, 4 (2015)

10. H. Zhou, Y. Yang, Ch. Zhang, D. Hu, European Journal of Environmental and Civil Engineering, 19, s1 (2015)

11. L.I. Ameur, G. Robin, M. Hattab, European Journal of Environmental and Civil Engineering, 20, 9 (2016)

12. S.H. Chow, C.D. O'loughlin, M.F. Randolph, Géotechnique, 64, 10 (2014)

13. S.S. Giroryan, Studies on soil mechanics. Thesis for a scientific degree of doctor of science (Moscow State University named after M.V. Lomonosov. Moscow, 1965)

14. A.A. Vovk, B.V. Zamyshlyaev, L.S. Yevterev, I.V. Belinsky, A.V. Mikhalyuk, Behavior of soils under the influence of impulse loads (Kiev: Naukova Dumka, 1984)

15. G.V. Rykov, A.M. Skobeev, Stress Measurement in Soils under Short-Term Loads (Moscow: Nauka, 1978)

16. K.S. Sultanov, Wave theory of seismic resistance of underground structures (Tashkent: "Fan", 2016)

17. K.S. Sultanov, J Appl Maths Mechs USA, 62, 3 (1998)

18. K.S. Sultanov, B.E. Khusanov, J Soil Mechanics \& Foundation Engineering USA, 38, 3 (2001)

19. K.S. Sultanov, A.A. Bakhodirov, Soil Mechanics and Foundation Engineering, 53, 2 (2016)

20. K.S. Sultanov, J.Appl. Maths.Mechs USA, 66, 1 (2002)

21. A.A. Bakhodirov, S.I. Ismailova, K.S. Sultanov, Journal of Applied Mathematics and Mechanics, 79, 6 (2015)

22. M.M. Mirsaidov, T.Z. Sultanov, Soil Mechanics and Foundation Engineering, 49, 6 (2013)

23. T.Z. Sultanov, D.A. Khodzhaev, M.M. Mirsaidov, Magazine of Civil Engineering, 45, 1 (2014) 